\title{
Procalcitonin Uptake and Effect on Antibiotics Stewardship: A Single Institution Experience
}

\author{
Hycienth Ahaneku ${ }^{1}$, MD DrPH, Chukwuemeka A. Umeh ${ }^{* 2}$, MD DrPH, Mike Cao ${ }^{3}$, MD, Bradley Kapten ${ }^{3}$, MD, \\ Baher Elhalwagi ${ }^{3}$, MD, Tiffany Egbe ${ }^{3}$, MD, Emmanuel Elueze ${ }^{4}, \mathrm{MD}, \mathrm{PhD}$ \\ ${ }^{1}$ Department of Hematology and Oncology, Beaumont Health Hospital Royal Oak, Michigan, USA \\ ${ }^{2}$ Department of Medicine, Hemet Valley Medical Center, Hemet, California, USA \\ ${ }^{3}$ Christus Good Shepherd Medical Center, Longview Texas USA \\ ${ }^{4}$ University of Texas Health Science Center, Tyler Texas, USA
}

Corresponding author: Chukwuemeka Anthony Umeh; Chukwuemeka.umeh@phh.ms; emmyumeh@yahoo.com

Received 18 August 2020;

Accepted 04 September 2020;

Published 15 September 2020

\begin{abstract}
Background: In the face of antibiotics resistance and adverse effects, emerging evidence suggests that procalcitonin guided therapy can help enhance appropriate use of antibiotics especially in patients with respiratory infections and sepsis. We seek to assess the uptake of procalcitonin among clinicians in a US hospital and assess its correlation with amount and duration of antibiotics use. We also seek to identify factors significantly associated with antibiotic use. Methodology: Retrospective cross-sectional study of patients with sepsis and COPD at a Medical Center in Texas USA. A total 48 COPD and 62 Sepsis patients were assessed. We collected demographic data such as age, weight, height, gender, and race/ethnicity. We also collected data on procalcitonin, number of antibiotics used, duration of antibiotics, and WBC levels at admission. We conducted bivariate analyses and logistic regression analyses to assess factor associated with procalcitonin, number of antibiotics used and duration of antibiotics. $\underline{\text { Results: }}$. We had 48 COPD patients and 62 sepsis patients in this study. Overall physicians ordered Procalcitonin on only $11.8 \%$ of patients. Procalcitonin was not significantly associated with antibiotics use. However, patients who had procalcitonin ordered had significantly lower WBC count than those without procalcitonin (10.1 vs. 12.9, p:0.026). Number of antibiotics was significantly associated with type of diagnosis (Sepsis $66.7 \%$ vs. COPD $50.7 \%, p=0.001$ ). In the logistic regression analysis, after adjusting for other variables patients with sepsis were significantly more likely to be on higher number antibiotics (OR 6.08, $\mathrm{p}<0.001$ ) and longer duration of antibiotics (OR 7.44, $\mathrm{p}$ $<0.001)$. Conclusion: Inappropriate use of antibiotics is a public health problem. Procalcitonin has been touted as a biomarker that is effective in reducing use of antibiotics. Our study showed a low utilization of Procalcitonin by physicians and that patients with lower WBC counts were more likely to have procalcitonin ordered. However, our study did not find any association between procalcitonin and the number and duration of antibiotics use.
\end{abstract}

\section{Introduction}

Since the discovery of penicillin by Flemming, antibiotics have played an important role in improving public health in the 20th century ${ }^{[1]}$. The last decades of the 20th century saw the proliferation of various antibiotics targeted against various bacterial organisms. However, in the last two decades we have seen the emergence of antibiotics resistance ${ }^{[1-3]}$. Antibiotics resistance has both mortality and economic implications in health care. The European Union (EU) estimates that 25,000 patients die yearly from infections from drug resistant bacteria and this is costing the EU about EUR1.5 billion of extra healthcare costs ${ }^{[4]}$. In the United States (US), the Center for Disease Control (CDC) estimates that antibiotics resistance contributes to more than two million infections and 23,000 deaths. In terms of health care costs, antibiotics resistance costs the US society about $\$ 2.9 \mathrm{M}$ every year ${ }^{[5,6]}$ The cost of antibiotics resistance also extends to lost productivity, prolonged morbidity and other social effects ${ }^{[5,6]}$.

The key driver of antimicrobial resistance is the selection pressure caused by antibiotic overuse ${ }^{[2]}$. Physicians often succumb to the pressure of the immediate needs of the patient at point of care leading to over-prescription of antibiotics ${ }^{[2,7,8]}$. Respiratory infections is the third leading cause of disease burden worldwide and is among the most common reasons for antibiotics prescription ${ }^{[9,10]}$. However, most of respiratory infections are of viral etiology and its sometimes difficult to clinically differentiate viral and bacterial causes. Several studies have shown that prescription of antibiotics for respiratory infections are often unnecessary or downright inappropriate. Even when necessary antibiotics treatments often exceed recommended duration ${ }^{[11,12]}$.

Inappropriate antibiotics prescription can lead to several effects - increased cost of care, increased risk or resistance (and its associated costs), increased risk of adverse effects (notably 
clostridium difficille infections) and prolonged exposure ${ }^{[3,13]}$. In view of these unwanted effects, several health care organizations and agencies including the CDC are pushing for responsible use of antibiotics - often described as antibiotics stewardship.

Physicians often rely on clinical judgment when making decisions on starting antibiotics therapy, and other adjuncts such as blood culture are not readily available ${ }^{[14]}$. Using biomarkers can be helpful in such circumstance. However, conventional biomarkers like complete blood count (CBC) and c-reactive protein (CRP) have their limitations as they are less sensitive and specific in detecting bacterial infections compared to procalcitonin ${ }^{[15,16]}$. Emerging evidence supports the use of procalcitonin to enhance the diagnosis of bacterial infections and thus improve antibiotics stewardship.

In inflammatory states, procalcitonin is released from many tissues leading to high levels in blood. Potent inducers of procalcitonin release include bacterial endotoxins (lipopolysaccharides), interleukin 6 (IL-6), IF-alpha and TNF-alpha ${ }^{[17,18]}$. Such increases are rarely seen with viral infections. It's been suggested that viruses release some cytokines that inhibit TNFalpha and IF-alpha ${ }^{[19-22]}$. The promise of procalcitonin is in its rapid rise in states of inflammation. It rises within 2-6 hours of inflammation which is faster than the release of CRP ${ }^{[23]}$. Procalcitonin has been compared to other markers like IL-6, white blood cell (WBC) and CRP and found to be a better discriminant infections compared to the others ${ }^{[15,24,25]}$.

Several studies have shown the benefit of procalcitonin guided therapy in reducing the duration of antibiotic use and antibiotics associated side effects without increasing the risk for mortality, recurrent infections, or treatment failures [26-31]. Procalcitonin guided therapy significantly reduced length of hospital stay and reduced the cost of pharmacy care and reduced the length of antibiotics exposure ${ }^{[32]}$. Contrary to majority of studies, a few studies did not show a difference in antibiotics use in procalcitonin guided therapy which could be as a result of differences in case mix, design, and setting ${ }^{[33]}$. In view of the campaign for proper antibiotic use, use of procalcitonin can help physicians in making antibiotic decisions in situations of diagnostic ambiguity. However, the uptake of procalcitonin by physicians when making antibiotics prescription decision is unclear. We seek to assess the uptake of procalcitonin among clinicians in a US hospital and assess its correlation with amount and duration of antibiotics use. We also seek to identify factors significantly associated with antibiotic use.

\section{Methodology}

This is a retrospective study of patients admitted to a university affiliated community hospital in Texas United States between May 1, 2018 and October 30, 2018. The Medical Center is a 425-bed major referral center for specialized care serving more than a population of 200,000 in East Texas, United States. Participants include adults greater than 18 years who were seen at the medical center during the time frame above.

\section{Data collection}

For data collection we engaged the services of hospital transcriptions specialists, who extracted relevant data from the electronic medical records and stored initially in excel file. Relevant data initially extracted include age, gender, ethnicity/race, diagnosis, and dates of admission. We then created a template for further data extraction. With the help of resident physicians, we factor in predicting septicemia, respiratory infections, and bacterial

further collected clinical data on each of the patients including antibiotics administered, duration of antibiotics, number of antibiotics, white blood count, hemoglobin, platelet count, creatinine, and procalcitonin orders. The data was subsequently deidentified and serial numbers assigned for the purpose of analyses.

\section{Statistical Analyses}

We first computed descriptive analysis on all variables collected including frequencies, means and standard deviation. We further did bivariate analysis comparing number of antibiotics administered and duration of antibiotics with procalcitonin orders, creatinine, age, diagnosis, and WBC. Significance testing was done using chi-squared test of proportions and independent t-test of means.

Finally, logistic regression analyses were done using number of antibiotics administered and duration of antibiotics as dependent variables. All other variables were included as independent variables. To facilitate the analyses, the dependent variables were each dichotomized. All analyses were done using the SPSS statistical package version 25 .

\section{Results}

A total of 540 patients that met the diagnostic code for sepsis and 94 patients with diagnostic code for chronic obstructive pulmonary disease (COPD) exacerbation were initially extracted by the hospital transcription specialist. From these a random sample of 62 sepsis patients and 48 COPD exacerbation patients were selected for extraction of more data. Thus, we had a total of 110 patients for analyses.

Descriptive analysis of the total patient population is presented in Table 1 below. The table shows that the average age of patients in our sample was 65 years. And when we dichotomized by age, $50.9 \%$ were 65 years and above. Our sample population was equally distributed gender wise; however, $79.1 \%$ were Caucasians. With an average sample weight of 85.57 kilograms and average height of 66.84 inches, the average body mass index (BMI) of the population was 29.7. Per interested variables, only $11.1 \%(\mathrm{~N}=13)$ of the sample had procalcitonin levels ordered.

Table 2 show the result of bivariate analysis between procalcitonin and other variables. There was no significant relationship between procalcitonin being ordered and each of the following variables: number of antibiotics, duration of antibiotics and type of diagnosis. However, patients who had procalcitonin ordered on them had significantly lower WBC count at admission compared to those without procalcitonin (10.1 vs. 12.9, p:0.026).

Table 1: Patient Characteristics

\begin{tabular}{|l|l|}
\hline Variable & Mean/\% \\
\hline Age & 65.53 \\
\hline Age (dichotomized) & \\
\hline$<=64$ & $49.10 \%$ \\
\hline$>=65$ & $50.90 \%$ \\
\hline Gender & \\
\hline Male & $50.90 \%$ \\
\hline Female & $49.10 \%$ \\
\hline Race & \\
\hline Black & $20.90 \%$ \\
\hline Caucasian & $79.10 \%$ \\
\hline Weight (kilograms) & 85.57 \\
\hline Height (Inches) & 66.84 \\
\hline Basic Labs & \\
\hline WBC & 12.69 \\
\hline
\end{tabular}




\begin{tabular}{|l|l|}
\hline HGB & 12.48 \\
\hline PLTS & 244.27 \\
\hline Creatinine & 1.21 \\
\hline Procalcitonin & \\
\hline Yes & $11.80 \%$ \\
\hline No & $88.20 \%$ \\
\hline Number of Antibiotics & 2.33 \\
\hline Number of Antibiotics & \\
\hline$<=2$ & $60 \%$ \\
\hline$>=3$ & $40 \%$ \\
\hline Duration of Antibiotics (Days) & 4.13 \\
\hline Duration of Antibiotics (Days) & \\
\hline$<=3$ & $58.20 \%$ \\
\hline$>=4$ & $41.80 \%$ \\
\hline Diagnosis & \\
\hline Sepsis & $43.60 \%$ \\
\hline COPD (Exacerbation) & $56.40 \%$ \\
\hline
\end{tabular}

Table 2: Bivariate Analysis on Procalcitonin

\begin{tabular}{|l|c|c|c|}
\hline & \multicolumn{3}{|c|}{ Procalcitonin Ordered } \\
\hline & Yes & No & P-value \\
\hline WBC & 10.1 & 12.9 & 0.026 \\
\hline Number of Antibiotics & & & 0.278 \\
\hline$<=2$ & $9.10 \%$ & $90.90 \%$ & \\
\hline$>=3$ & $15.90 \%$ & $84.10 \%$ & \\
\hline Duration of Antibiotics (Days) & & & 0.794 \\
\hline$<=3$ & $12.50 \%$ & $87.50 \%$ & \\
\hline$>=4$ & $10.90 \%$ & $89.10 \%$ & \\
\hline Diagnosis & & & 0.689 \\
\hline Sepsis & $10.40 \%$ & $89.60 \%$ & \\
\hline COPD (Exacerbation) & $12.90 \%$ & $87.10 \%$ & \\
\hline
\end{tabular}

Table 3 shows result of bivariate analysis on number of antibiotics. Here again procalcitonin was not significantly associated with number of antibiotics used. However, patients with three or more antibiotics had significantly higher mean creatinine level compared to patients with two or less antibiotics prescribed. Additionally, Table 3 reveals that sepsis patients were more likely to be on greater number of antibiotics compared to patients with COPD exacerbation (66\% vs. $50 \%$, p <0.001).

Table 3: Bivariate Analysis on Number of Antibiotics Used

\begin{tabular}{|l|c|c|c|}
\hline & \multicolumn{3}{|c|}{ Number of Antibiotics } \\
\hline & $<=\mathbf{2}$ & $>\mathbf{3}$ & P-value \\
\hline WBC & 11.5 & 14.2 & 0.055 \\
\hline Creatinine (mg/dl) & 1.01 & 1.51 & 0.027 \\
\hline Age & 64.3 & 67.1 & 0.207 \\
\hline Hemoglobin (g/dl) & 12.6 & 12.3 & 0.565 \\
\hline Procalcitonin checked & & & 0.278 \\
\hline Yes & $46.20 \%$ & $53.80 \%$ & \\
\hline No & $61.90 \%$ & $38.10 \%$ & \\
\hline $\begin{array}{l}\text { Duration of Antibiotics } \\
\text { (Days) }\end{array}$ & & & $<0.001$ \\
\hline$<=3$ & $78.10 \%$ & $21.90 \%$ & \\
\hline$>=4$ & $34.80 \%$ & $65.20 \%$ & \\
\hline Diagnosis & & & $<0.001$ \\
\hline Sepsis & $33.30 \%$ & $66.70 \%$ & \\
\hline COPD (Exacerbation) & $50.00 \%$ & $50.00 \%$ & \\
\hline
\end{tabular}

Table 4 below shows the results of bivariate analyses on duration of antibiotics use. Here again diagnosis was significantly associated with duration of antibiotics. Patients with sepsis were more likely to be on antibiotics for a longer duration compared to

patients with COPD exacerbation $(66.7 \%$ vs. $22.6 \%$, p < 0.001$)$. Procalcitonin did not show any significant association with duration of antibiotics.

Table 4: Bivariate Analysis on Duration of Antibiotics

\begin{tabular}{|l|c|c|c|}
\hline & \multicolumn{3}{|c|}{ Duration of Antibiotics (days) } \\
\hline & $<=\mathbf{3}$ & $>=\mathbf{4}$ & P-value \\
\hline WBC & 12.3 & 12.9 & 0.64 \\
\hline Creatinine (mg/dl) & 1.05 & 1.25 & 0.113 \\
\hline Age & 65 & 66 & 0.597 \\
\hline Hemoglobin (g/dl) & 12.65 & 12.25 & 0.433 \\
\hline Procalcitonin ordered & & & 0.747 \\
\hline YES & $61.50 \%$ & $38.50 \%$ & \\
\hline NO & $57.70 \%$ & $42.30 \%$ & \\
\hline Number of Antibiotics & & & $<0.001$ \\
\hline$<=2$ & $75.80 \%$ & $24.20 \%$ & \\
\hline$>=3$ & $31.80 \%$ & $68.20 \%$ & \\
\hline Diagnosis & & & $<0.001$ \\
\hline Sepsis & $33.30 \%$ & $66.70 \%$ & \\
\hline COPD (Exacerbation) & $77.40 \%$ & $22.60 \%$ & \\
\hline
\end{tabular}

Tables 5 and 6 show results of logistic regression analyses with number and duration of antibiotics as dependent variables respectively. For both models, after adjusting for age, creatinine level, WBC count and procalcitonin, patients with sepsis were significantly more likely to be on higher number (OR 6.08, p $<0.001$ ) and duration (OR 7.44, p < 0.001) of antibiotics. Procalcitonin was not significantly associated with any of these outcomes.

Table 5: Logistic Regression Analysis Predicting Likelihood of Using >=3 Antibiotics

\begin{tabular}{|l|c|c|c|c|}
\hline Variable & OR & $\begin{array}{c}\text { Lower } \\
\text { CI }\end{array}$ & $\begin{array}{c}\text { Upper } \\
\text { CI }\end{array}$ & $\begin{array}{c}\text { P- } \\
\text { value }\end{array}$ \\
\hline Age & 1.02 & 0.98 & 1.06 & 0.403 \\
\hline Creatinine & 1.72 & 0.74 & 3.99 & 0.205 \\
\hline WBC & 1.04 & 0.97 & 1.12 & 0.243 \\
\hline Procalcitonin (YES) & 4.03 & 1.00 & 16.24 & 0.050 \\
\hline Diagnosis (SEPSIS) & 6.08 & 2.32 & 15.91 & 0.000 \\
\hline
\end{tabular}

Table 6: Logistic Regression Analysis Predicting Likelihood of $>=4$ days of Antibiotics

\begin{tabular}{|l|c|c|c|c|}
\hline Variable & OR & $\begin{array}{c}\text { Lower } \\
\text { CI }\end{array}$ & $\begin{array}{c}\text { Upper } \\
\text { CI }\end{array}$ & $\begin{array}{c}\text { P- } \\
\text { value }\end{array}$ \\
\hline Age & 1.00 & 0.96 & 1.03 & 0.865 \\
\hline Creatinine & 1.07 & 0.50 & 2.31 & 0.854 \\
\hline WBC & 0.97 & 0.91 & 1.04 & 0.365 \\
\hline Procalcitonin (YES) & 0.88 & 0.22 & 3.49 & 0.857 \\
\hline Diagnosis (SEPSIS) & 7.44 & 2.84 & 19.51 & 0.000 \\
\hline
\end{tabular}

\section{Discussion}

In our study, we assessed the uptake of procalcitonin by physicians in the management of sepsis and COPD exacerbation. Of the 110 patients assessed, only $11.8 \% \quad(\mathrm{~N}=13)$ of the patients had procalcitonin ordered on their admission. The results reveal a low physicians' uptake of procalcitonin as a tool for managing sepsis and COPD in the hospital setting. Not much studies have been done to assess the use of procalcitonin in managing sepsis and COPD patients. The low physicians' uptake of procalcitonin test in this study may be because procalcitonin test was introduced in the hospital less than six months prior to this study and studies have 
shown that adoption of new innovations could be slow in all industries including health care ${ }^{[34]}$.

We also assessed the relationship between procalcitonin and antibiotics prescription viz number of antibiotics and duration of antibiotics. In both the chi squared analysis and logistic regression analyses ordering a procalcitonin test was not associated with number of antibiotics used, duration of antibiotic use and type of diagnosis (SEPSIS or COPD). This finding differs from some studies that showed that procalcitonin reduced the number and duration of antibiotics use ${ }^{[26-32]}$. However, similar to our finding, a few studies did not show a difference in antibiotics use in procalcitonin guided therapy ${ }^{[33]}$.

Furthermore, our analyses show that patients with lower WBC counts were more likely to have procalcitonin ordered. The mean WBC in those that procalcitonin was ordered was 10 compared with $13(\mathrm{p}=0.02)$ in those it was not ordered (Table 2). This suggests that clinicians are more likely to order procalcitonin when they have in doubt about whether there is an infection such as in patients with lower WBC and probably more likely to start antibiotics without ordering procalcitonin when they have a strong suspicion of an infection e.g. higher WBC. This agrees with previous studies that have shown higher odds of antibiotics prescription when clinicians have a high clinical suspicion of infection ${ }^{[14]}$.

Beyond procalcitonin, our study found that prescription behavior (number of antibiotics and duration of antibiotics) were significantly associated with type of diagnosis. This may not be surprising as physicians are likely to start antibiotics with high WBC and when any patient meets sepsis criteria. In our study, patients with sepsis were significantly more likely to be on higher number (OR 6.08, p <0.001) and duration (OR 7.44, p < 0.001) of antibiotics.

There are some limitations in our study. First our study had a small sample size which could have affected the ability to detect a difference between the procalcitonin and non-procalcitonin groups. Although we did not perform a rigorous sensitivity analyses, an anecdotal comparison of our sample size with other studies suggests our small numbers could have underpowered our study to detect a significant difference. Secondly, our study had a retrospective and cross-sectional design; thus, it is more prone to threats to internal validity compared to prospective randomized controlled trials.

\section{Conclusion}

Inappropriate use of antibiotics is a public health problem. Procalcitonin has been touted as a biomarker that is effective in reducing use of antibiotics. Our study showed a low utilization of procalcitonin by physicians and that patients with lower WBC counts were more likely to have procalcitonin ordered. However, our study did not find any association between procalcitonin and the number and duration of antibiotics use.

\section{Acknowledgements}

We very much appreciate the help of Norman Jordan and Katherine Smith in helping to extract the data for this study.

\section{References}

[1] Aminov RI. A brief history of the antibiotic era: lessons learned and challenges for the future. Front Microbiol.
2010; 1:134. Published 2010 Dec 8. doi:10.3389/fmicb.2010.00134

[2] Wise R, Hart T, Cars O, et al. Antimicrobial resistance is a major threat to public health. BMJ. 1998; 317:609-610. doi: 10.1136/bmj.317.7159.609.

[3] Huttner A, Harbarth S, Carlet J, et al. Antimicrobial resistance: a global view from the 2013 World Healthcare-Associated Infections Forum. Antimicrob Resist Infect Control. 2013; 2:31. Published 2013 Nov 18. doi:10.1186/2047-2994-2-31.

[4] European Centre for Disease Prevention Control/European Medicines Agency Joint Working Group (ECDC/EMEA) (2009). The Bacterial Challenge: Time to React. Accessed on February 23, 2020 from https://www.ecdc.europa.eu/sites/default/files/media/en/p ublications/Publications/0909_TER_The_Bacterial_Chal lenge_Time_to_React.pdf

[5] Shrestha P, Cooper BS, Coast J, et al. Enumerating the economic cost of antimicrobial resistance per antibiotic consumed to inform the evaluation of interventions affecting their use. Antimicrob Resist Infect Control. 2018;7:98. Published 2018 Aug 9. doi:10.1186/s13756018-0384-3.

[6] Centers for Disease Control and Prevention. Antibiotic Resistance Threats in the United States, 2013. Atlanta, GA: Centers for Disease Control and Prevention. Accessed on February 23, 2020 from https://www.cdc.gov/drugresistance/pdf/ar-threats-2013508.pdf

[7] Macfarlane J, Holmes W, Macfarlane R, Britten N. Influence of patients' expectations on antibiotic management of acute lower respiratory tract illness in general practice: questionnaire study. BMJ. 1997;2(7117):1211-1214. doi: 10.1136/bmj.315.7117.1211.

[8] Dunagan WC, Woodward RS, Medoff G, et al. Antimicrobial misuse in patients with positive blood cultures. Am J Med. 1989; 87:253-259.

[9] Global Health Estimates 2016. Deaths by Cause, Age and Sex by Country and by Region, 2000 - 2016. Geneva, World Health Organization, 2018.

[10] Harris AM, Hicks LA, Qaseem A, High Value Care Task Force of the American College of Physicians and for the Centers for Disease Control and Prevention. Appropriate Antibiotic Use for Acute Respiratory Tract Infection in Adults: Advice for High-Value Care from the American College of Physicians and the Centers for Disease Control and Prevention. Ann Intern Med 2016; 164:425.

[11] Fleming-Dutra KE, Hersh AL, Shapiro DJ, et al. Prevalence of Inappropriate Antibiotic Prescriptions Among US Ambulatory Care Visits, 2010-2011. JAMA 2016; 315:1864.

[12] Shapiro DJ, Hicks LA, Pavia AT, Hersh AL. Antibiotic prescribing for adults in ambulatory care in the USA, 2007-09. J Antimicrob Chemother 2014; 69:234.

[13] CDC. Core Elements of Hospital Antibiotic Stewardship Programs. Accessed on February 23, 2020 from https://www.cdc.gov/antibioticuse/healthcare/implementation/core-elements.html\#

[14] McKay R, Mah A, Law MR, et al. Systematic Review of Factors Associated with Antibiotic Prescribing for Respiratory Tract Infections. Antimicrob Agents Chemother 2016; 60:4106. 
[15] Müller B, Harbarth S, Stolz D, Bingisser R, Mueller C, Leuppi J, Nusbaumer C, Tamm M, Christ-Crain M. Diagnostic and prognostic accuracy of clinical and laboratory parameters in community-acquired pneumonia. BMC infectious diseases. 2007 Dec $1 ; 7(1): 10$.

[16] Simon L, Gauvin F, Amre DK, Saint-Louis P, Lacroix J. Serum procalcitonin and C-reactive protein levels as markers of bacterial infection: a systematic review and meta-analysis. Clinical infectious diseases. $2004 \mathrm{Jul}$ 15;39(2):206-17.

[17] Müller F, Christ-Crain M, Bregenzer T, et al; ProHOSP Study Group. Procalcitonin levels predict bacteremia in patients with community-acquired pneumonia: a prospective cohort trial. Chest. 2010; 138 (1): 121 - 129.

[18] Schuetz P, Christ-Crain M, Albrich W, Zimmerli W, Mueller B; ProHOSP Study Group. Guidance of antibiotic therapy with procalcitonin in lower respiratory tract infections: insights into the ProHOSP study. Virulence; $2010 ; 1(2): 88-92$.

[19] Christ-Crain M, Müller B. Procalcitonin in bacterial infections - hype, hope, more or less? Swiss Med Wkly. 2005; 135 (31-32): 451 - 460.

[20] Christ-Crain M, Müller B. Biomarkers in respiratory tract infections: diagnostic guides to antibiotic prescription, prognostic markers and mediators. Eur Respir J. 2007; 30 (3): 556- 573.

[21] Linscheid P, Seboek D, Zulewski H, Keller U, Müller B. Autocrine/paracrine role of inflammation-mediated calcitonin gene-related peptide and adrenomedullin expression in human adipose tissue. Endocrinology. 2005; 146(6): 2699- 2708.

[22] Schuetz P, Christ-Crain M, Müller B. Procalcitonin and other biomarkers to improve assessment and antibiotic stewardship in infections-hope for hype? Swiss Med Wkly. 2009; 139 (23-24): 318 - 326.

[23] Dandona P, Nix D, Wilson MF, Aljada A, Love J, Assicot M, Bohuon CL. Procalcitonin increase after endotoxin injection in normal subjects. The Journal of Clinical Endocrinology \& Metabolism. 1994 Dec 1;79(6):1605-8.

[24] Tsalik EL, Jaggers LB, Glickman SW, et al. Discriminative value of inflammatory biomarkers for suspected sepsis. J Emerg Med. 2011;43(1):97-106. doi: 10.1016/j.jemermed.2011.05.072.

[25] Nargis W, Ibrahim M, Ahamed BU. Procalcitonin versus C-reactive protein: Usefulness as biomarker of sepsis in ICU patient. Int J Crit Illn Inj Sci. 2014;4(3):195-199. doi:10.4103/2229-5151.141356.

[26] Schuetz P, Christ-Crain M, Thomann R, et al. Effect of procalcitonin-based guidelines vs standard guidelines on antibiotic use in lower respiratory tract infections: the ProHOSP randomized controlled trial. JAMA 2009; 302:1059.

[27] Schuetz P, Wirz Y, Sager R, et al. Procalcitonin to initiate or discontinue antibiotics in acute respiratory tract infections. Cochrane Database Syst Rev 2017; 10:CD007498.

[28] Sager R, Kutz A, Mueller B, Schuetz P. Procalcitoninguided diagnosis and antibiotic stewardship revisited. BMC medicine. 2017 Dec;15(1):15.

[29] Mathioudakis AG, Chatzimavridou-Grigoriadou V, Corlateanu A, Vestbo J. Procalcitonin to guide antibiotic administration in COPD exacerbations: a meta-analysis. Eur Respir Rev 2017; 26.

[30] Stolz D, Christ-Crain M, Bingisser R, et al. Antibiotic treatment of exacerbations of COPD: a randomized, controlled trial comparing procalcitonin-guidance with standard therapy. Chest 2007; 131:9.

[31] Corti C, Fally M, Fabricius-Bjerre A, et al. Point-of-care procalcitonin test to reduce antibiotic exposure in patients hospitalized with acute exacerbation of COPD. Int J Chron Obstruct Pulmon Dis 2016; 11:1381.

[32] Balk RA, Kadri SS, Cao Z, Robinson SB, Lipkin C, Bozzette SA. Effect of Procalcitonin Testing on Healthcare Utilization and Costs in Critically Ill Patients in the United States. Chest. 2016;151(1):23-33. doi: 10.1016/j.chest.2016.06.046.

[33] Huang DT, Yealy DM, Filbin MR, Brown AM, Chang $\mathrm{CH}$, Doi $\mathrm{Y}$, et al; ProACT Investigators.Procalcitoninguided use of antibiotics for lower respiratory tract infection. N Engl J Med. 2018.

[34] Berwick DM. Disseminating innovations in health care. Jama. 2003 Apr 16;289(15):1969-75. 